\title{
La gestion de la reproduction caprine au travers des femelles vendues à différents points de vente de Lubumbashi en République Démocratique du Congo
}

\author{
Ngona I.A. ${ }^{1}$, Masenga P. ${ }^{2}$, Khang'Maté A.B. ${ }^{1}$ \\ 1 Service de Reproduction, Obstétrique et Insémination Artificielle, Faculté de Médecine Vétérinaire, Université de \\ Lubumbashi, RDC \\ 2 Faculté de Médecine Vétérinaire, Université de Lubumbashi, RDC \\ Corresponding author email: ingona@yahoo.fr
}

Original submitted in on 24th March 2015. Published online at www.m.elewa.org on $31^{\text {st }}$ July 2015 http://dx.doi.org/10.4314/jab.v91i1.4

\section{RESUME}

Objectif : L'objectif consistait à préciser l'importance des pertes de gestation, relever le niveau de gestion de la reproduction caprine et spécifier la rentabilité des caprins $(n=5415)$ exposés à différents points de vente de Lubumbashi d'avril à août 2011.

Méthodologie et résultats: La collecte des données reposait sur la détermination de la provenance des animaux par interview des vendeurs des différents points de vente. Le nombre de mâles et de femelles procédait du comptage effectué une fois par semaine à chaque point de vente. Les femelles, choisies au hasard, identifiées selon la dentition, étaient soumises au constat de gestation par échographie. Pour l'étude descriptive, le traitement des données reposait sur des calculs des proportions et des comparaisons des moyennes. L'étude relationnelle s'appuyait sur le test de khi carré. L'étude descriptive avait présenté des proportions mensuelles comprises entre 15 et $23 \%$ des caprins étudiés avec de pic (mai) et de valeur basale (août). Le constat de gestation par échographie avait présenté de femelles non gestantes (51\%) et gestantes (49\%). L'étude relationnelle avait montré que les effectifs des mâles (53\%) et femelles (47\%) observés étaient dépendants du sexe $(p<0,05)$. La disponibilité des femelles aux points de vente était fonction de mois et les différences entre les effectifs des femelles aux différents points de vente pour chaque mois étaient significatives $(p<0,05)$. II $n$ y avait pas de dépendance entre l'âge des femelles examinées et le résultat de l'échographie $(p>0,05)$. Néanmoins, l'effectif des femelles gestantes observées était fonction de l'âge $(p<0,05)$. Conclusion et application des résultats : Ces travaux avaient relevé une mauvaise gestion de la reproduction caprine. Toutefois, au regard des ventes mensuelles, l'élevage caprin pourrait être rentable dans la ville à Lubumbashi. Une assistance en conduite d'élevage pour pallier les pertes de gestation et mettre à profit la prolificité de la chèvre serait un atout pour l'élevage familial à Lubumbashi.

Mot-clés : caprins, points de vente, femelles gestantes, gestion de la reproduction, Lubumbashi. 


\section{ABSTRACT}

\section{Goat breeding management of females sold in Lubumbashi markets in the Democratic Republic of Congo}

Objective: The objective was to clarify the importance of pregnancy loss, raise the level of management goat reproduction and specify the profitability of goats $(n=5415)$ exposed to different Lubumbashi outlets from April to August 2011.

Methodology and results: The data collection was based on the determination of the origin of animals by interviewing the sellers at the various points of sale. The number of males and females was done by the counting made once a week in every point of sale. Females, chosen at random, identified according to the set of teeth, were subjected to ultrasound to determine gestation. For the descriptive study, the data processing was based on calculations of the proportions and the comparisons of the averages. The relational study leaned on the test of chi-square. The descriptive study presented the monthly proportions between 15 and $23 \%$ of goats studied with peak (in May) and with basal value (in August). The report of gestation by ultrasound had presented females non-pregnant $(51 \%)$ and pregnant $(49 \%)$. Relational study showed that the number of males $(53 \%)$ and females $(47 \%)$ observed were dependent on sex $(p<0.05)$. The availability of female at outlets was linked to months and differences between the numbers of females at different outlets for each month were significant $(p<0.05)$. There was no dependency between age of females examined and results of ultrasonography $(p>0.05)$. Nevertheless, the number of pregnant females was observed according to age ( $p$ $<0.05$ ).

Conclusion and application of results: This work found mismanagement of the goat reproduction. However, with regard to the monthly sales, the goat breeding could be profitable in the city of Lubumbashi. Livestock management assistance to offset losses of gestation and leverage prolificacy of the goat would be an asset to Lubumbashi family farms

Keywords: goats, points of sale, pregnant females, management of the reproduction, Lubumbashi.

\section{INTRODUCTION}

Dans les pays en développement, les carences en protéines animales rendent les populations vulnérables aux maladies spécialement les jeunes et les enfants. Pour pallier les carences, la chèvre occupe une place de choix par sa résistance aux maladies, sa capacité de reproduction et ses faibles coûts d'exploitation (Zarrouk et al., 2001). La viande de chèvre est adoptée universellement pour son arôme, sa saveur et sa tendreté. Les modalités de sa consommation sont largement influencées par les traditions culturelles et les conditions socioéconomiques (Ngona, 2008). L'élevage caprin est appelé à se développer dans les pays dits émergents. Il joue un rôle essentiel dans l'économie rurale comme source d'emplois, la viande sert d'aliment et de revenus surtout pour les petits éleveurs et offre des perceptives socioculturelles (Ngona, 2008). Bien plus, on observe un taux de fertilité élevé, un intervalle entre mise- bas moyen mais pouvant être amélioré rapidement par les conditions alimentaires et d'exploitations favorables du fait que les petits ruminants sont susceptibles de se reproduire toute l'année. L'élevage des caprins sous les tropiques ne présente pas une bonne gestion de la reproduction. En effet, l'éleveur ne parvient pas à établir un bon suivi mensuel de reproduction. Cet élevage ne jouit pas de la pratique de bilan de reproduction en identifiant les critères de sélection des animaux au travers de constat précoce ou tardif de gestation, en distinguant dans un premier temps la fertilité ou la fécondité et en interprétant dans un second temps les paramètres de fécondité et de fertilité . II s'en suit une forte proportion des femelles gestantes qui sont abattues aux marchés sans pour autant se baser sur les critères objectifs de reproduction. En effet, 1 chèvre sur 2 abattues à Lubumbashi est gestante (Ngona, 2008, Ngona et al., 2012). A Lubumbashi, la chèvre " espèce tout terrain ", est un atout pour la survie de la famille face aux graves problèmes de sécurité alimentaire dans une population dont $71 \%$ est sous alimentée. Elle constitue un véritable «compte 

différents points de vente de Lubumbashi en RDC.

d'épargne sur pied » dans un contexte où $80 \%$ de la population vit en dessous du seuil de pauvreté, soit avec moins de 1 dollar par jour (Vandenput, 2006). L'objectif du présent travail a consisté à préciser

\section{MILIEU, MATERIEL ET METHODES}

Milieu : Nos investigations s'étaient déroulées dans la ville de Lubumbashi auprès des vendeurs du Marché L.D. Kabila $\left(11^{\circ} 40^{\prime} 18,5^{\prime \prime}\right.$, latitude Sud et $027^{\circ} 29^{\prime} 21,4^{\prime \prime}$ longitude Est à $1180 \mathrm{~m}$ d'altitude), du Camp VANGU $\left(11^{\circ} 41^{\prime} 39,8^{\prime \prime}\right.$ latitude Sud et $027^{\circ} 29^{\prime} 28,8^{\prime \prime}$ longitude Està $1242 \mathrm{~m}$ d'altitude) et du Rail de KAMALONDO $\left(11^{\circ} 41^{\prime} 07,0^{\prime \prime}\right.$, latitude Sud et $027^{\circ} 29^{\prime} 15,0^{\prime \prime}$ longitude Està $1252 \mathrm{~m}$ d'altitude) d'avril à août 2011. l'importance des pertes de gestation, relever le niveau de gestion de la reproduction caprine à Lubumbashi et à spécifier la rentabilité des caprins à Lubumbashi.

Animaux : Les animaux examinés étaient des caprins (Capra hircus) provenant essentiellement de la Zambie et des fermes environnantes de la ville de Lubumbashi. L'effectif était variable selon la période de récolte des données; la taille totale de notre échantillon était composée de 5416 caprins mâles et femelles (Tableau 1).

Tableau 1 : Effectif des caprins aux différents points de vente

\begin{tabular}{llllll}
\hline & Avril & Mai & Juin & Juillet & Août \\
\hline Marché L.D. KABILA & 362 & 403 & 406 & 433 & 224 \\
Camp VANGU & 376 & 405 & 424 & 364 & 289 \\
Rail KAMALONDO & 286 & 408 & 395 & 367 & 274 \\
Total & 1024 & 1216 & 1225 & 1164 & 787 \\
Moyenne & 512 & 608 & 613 & 582 & 394 \\
Fréquence $\%$ & 19 & 22 & 23 & 21 & 15 \\
\hline
\end{tabular}

\section{Matériel et Méthodes}

Détermination de la provenance des animaux: L'information relative à la provenance des caprins était collectée auprès des vendeurs des différents points de vente mieux identifiés à l'aide de GPS de marque GARMIN. La méthode d'interview (pour déterminer la provenance des sujets concernés) avait présenté beaucoup de difficultés parce que les vendeurs des différents points de vente présenté ci-haut ne savaient pas déterminer la provenance des animaux par rapport aux fermes ou de préciser l'origine des bêtes. Le comptage se faisait une fois par semaine à chaque point de vente, soit quatre fois par mois en déterminant le nombre de mâles et de femelles. Ainsi pour le comptage, les cornes étaient marquées à la craie blanche. En effet, les animaux n'étaient pas identifiés par des boucles d'oreilles ou par d'autres signes distinctifs.

Constat de gestation par échographie : Les femelles, choisies au hasard, étaient identifiées selon la dentition pour déterminer leur âge respectif (Tableau 2) La fréquence des examens était bimensuelle et s'effectuaient à l'aide d'un échographe de marque 100 Falco Vet Pie Médical à sonde sectorielle de $50 \mathrm{MHz}$ (Photo 1). 

différents points de vente de Lubumbashi en RDC.

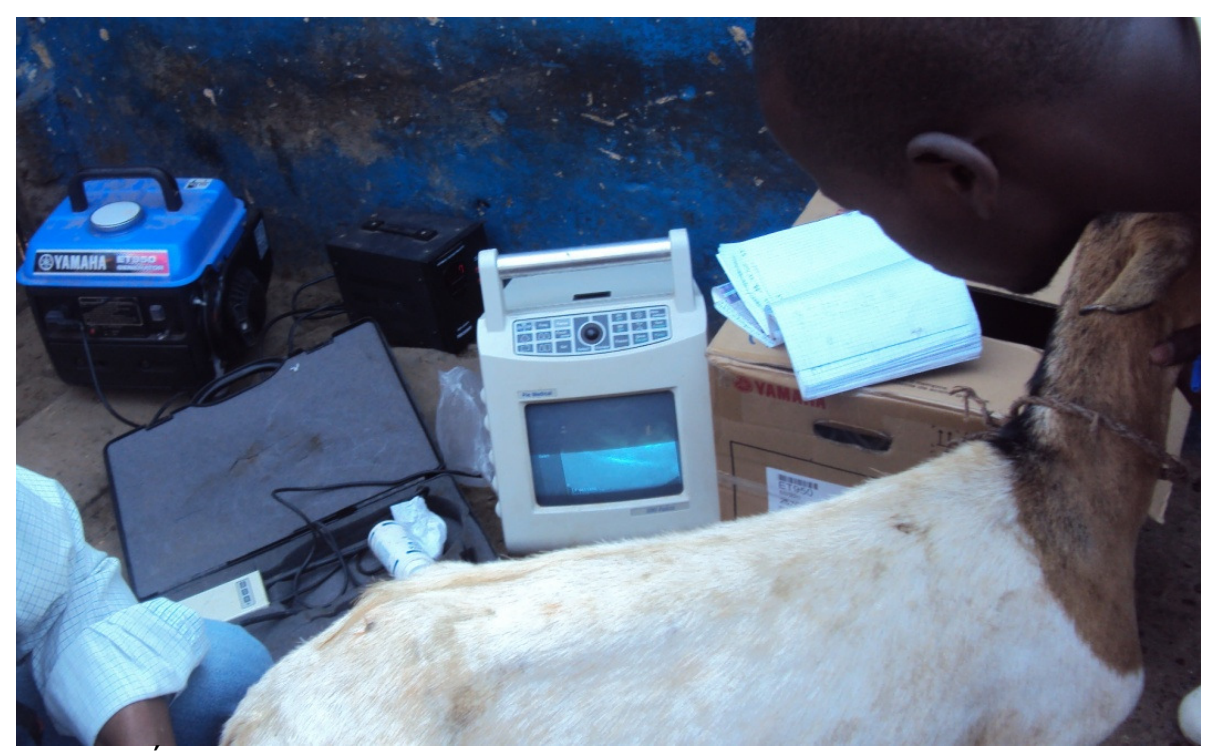

Photo 1. Échographe de marque 100 Falco Vet Pie Médical à sonde sectorielle

Tableau 2 : Détermination de l'âge correspondant (mois) selon les stades dentaires (Ngona, 2008)

\begin{tabular}{llllll}
\hline Dentition & Age & Dentition & Age & Dentition & Age \\
\hline Dents caduques & & Dents de remplacement & & Nivellement & \\
\hline 0 dent de lait & 0,1 & 2 dents adultes & 15 & 8 dents adultes entamées & 84 \\
2 dents de lait & 0,2 & 4 dents adultes & 21 & 8 dents adultes usées & 108 \\
4 dents de lait & 0,4 & 6 dents adultes & 26 & & \\
6 dents de lait & 0,4 & 8 dents adultes & 36 & & \\
8 dents de lait & 7 & & & & \\
\hline
\end{tabular}

Analyse statistique: Pour l'étude descriptive, le traitement des données procédait de la comparaison de deux ou plusieurs échantillons, il s'en suivait des calculs

\section{RESULTATS}

Effectif des mâles et femelles : L'étude descriptive avait permis de constater que la proportion des mâles et femelles exposés aux points de vente représentait respectivement 53 et $47 \%$. Au cours de la période de collecte des données ; les animaux étaient exposés dans des proportions variant entre 787 et 1225 individus, soit un pourcentage mensuel compris entre 15 et $23 \%$ des proportions et des comparaisons des moyennes. Au cours de l'étude relationnelle, le traitement des données reposait sur le test de khi carré.

(Tableau 3). Les mâles exposés aux points de vente présentaient des effectifs compris entre 171 et 202 individus (Tableau 5). L'étude relationnelle avait montré que les effectifs des mâles (53\%) et femelles (47\%) observés aux différents points de vente (Tableau 4) étaient dépendants du sexe $(p<0,05)$.

Tableau 3 : Effectif total des caprins selon les mois aux différents points de vente

\begin{tabular}{lllll}
\hline & Mâles & Femelles & Total & $(\%)$ \\
\hline Avril & 552 & 472 & 1024 & 19 \\
Mai & 592 & 624 & 1216 & 22 \\
Juin & 645 & 580 & 1225 & 23 \\
Juillet & 640 & 524 & 1164 & 21 \\
Août & 416 & 371 & 787 & 15 \\
Total & 2845 & 2571 & 5416 & 100 \\
$\%$ & 53 & 47 & 100 & \\
\hline
\end{tabular}


Ngona et al. J. Appl. Biosci. La gestion de la reproduction caprine au travers des femelles vendues à différents points de vente de Lubumbashi en RDC.

Tableau 4 : Effectif total des mâles et femelles observés aux différents points de vente

\begin{tabular}{lllll}
\hline & Marché L.D. KABILA & Camp VANGU & Rail KAMALONDO & $\%$ \\
\hline Mâles & 979 & 1010 & 856 & 53 \\
Femelles & 849 & 848 & 874 & 47 \\
Total & 1828 & 1858 & 1730 & 100 \\
\hline
\end{tabular}

Tableau 5 : Effectif des mâles observés par mois selon les différents points de vente

\begin{tabular}{lllllll}
\hline & Avril & Mai & Juin & Juillet & Août & Moyenne \\
\hline Marché L.D. KABILA & 198 & 219 & 202 & 244 & 116 & 196 \\
Camp VANGU & 204 & 205 & 229 & 214 & 158 & 202 \\
Rail KAMALONDO & 150 & 168 & 214 & 182 & 142 & 171 \\
Total & 552 & 592 & 645 & 640 & 416 & 569 \\
Moyenne & 184 & 197 & 215 & 213 & 139 & \\
$\%$ & 19 & 21 & 23 & 22 & 15 & \\
\hline
\end{tabular}

Effectif des femelles aux différents points de vente : L'étude descriptive avait montré pour les caprins exposés aux différents points de vente le pic en mai (132 à 179 caprins exposés) et les valeurs basales en août (71 à 126 caprins exposés) (Fig. 2). Les moyennes mensuelles avaient présenté les mêmes variations. En effet, les mois de mai et d'août avaient présenté des proportions de caprins exposés aux points de vente respectivement élevées ( 52 caprins par jour) ( $24 \%$ ) et faibles ( 31 caprins par jour) (14\%) (Tableau 6, 7). Au travers de l'étude relationnelle, il était observé que la disponibilité des femelles aux différents points de vente était fonction de mois et que les différences entre les effectifs des femelles aux différents points de vente pour chaque mois étaient significatives $(p<0,05)$.

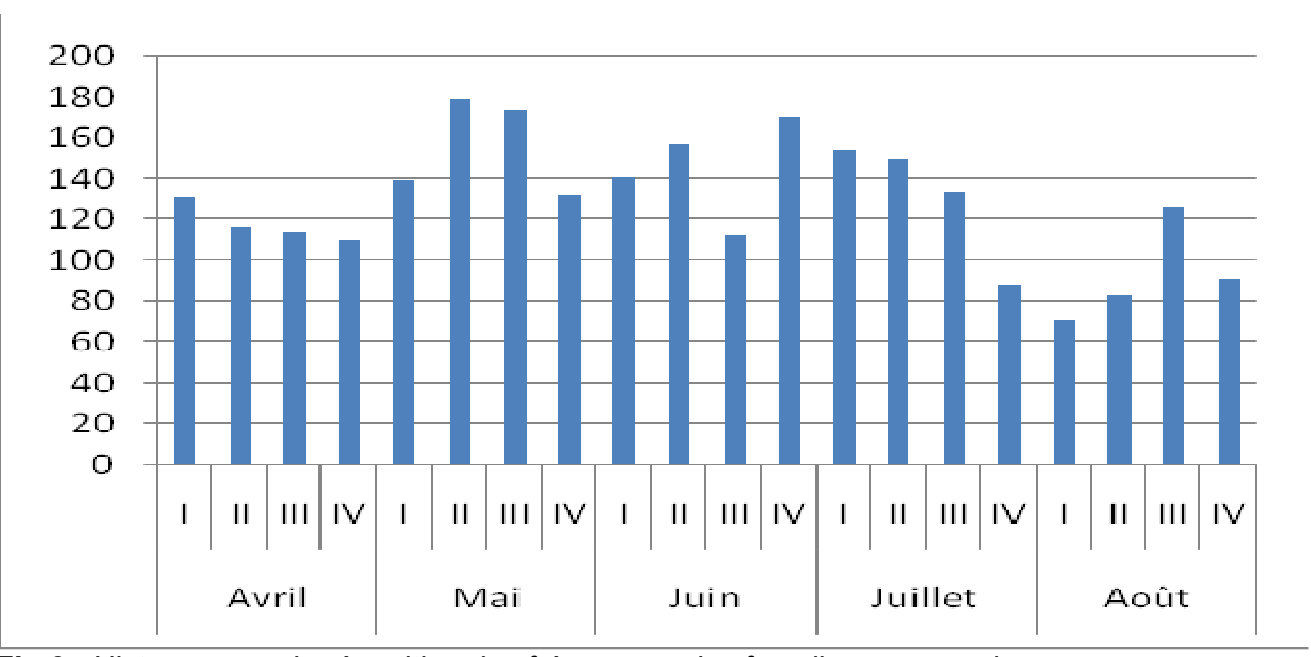

Fig.2 : Histogramme de répartition des fréquences des femelles par semaine

Tableau 6: Effectif moyen des femelles observées journellement selon les semaines aux différents points de vente

\begin{tabular}{lllll}
\hline & Marché L.D. KABILA & Camp VANGU & Rail KAMALONDO & Moyenne \\
\hline avril & 41 & 43 & 34 & 39 \\
mai & 46 & 50 & 60 & 52 \\
juin & 51 & 49 & 45 & 48 \\
juillet & 47 & 38 & 47 & 44 \\
août & 27 & 33 & 33 & 31 \\
\hline
\end{tabular}


Ngona et al. J. Appl. Biosci. La gestion de la reproduction caprine au travers des femelles vendues à différents points de vente de Lubumbashi en RDC.

Tableau 7 : Effectif des femelles observées par mois aux différents points de vente

\begin{tabular}{llllll}
\hline & Avril & Mai & Juin & Juillet & Août \\
\hline Marché L.D. KABILA & 164 & 184 & 204 & 189 & 108 \\
Camp VANGU & 172 & 200 & 195 & 150 & 131 \\
Rail KAMALONDO & 136 & 240 & 181 & 185 & 132 \\
Total & 472 & 624 & 580 & 524 & 371 \\
Moyenne & 157 & 208 & 193 & 175 & 124 \\
$\%$ & 18 & 24 & 23 & 20 & 14 \\
\hline
\end{tabular}

Effectif des femelles gestantes : L'étude descriptive au terme du constat de gestation par échographie avait présenté $51 \%$ de femelles non gestantes et $49 \%$ de femelles gestantes. Les femelles dont l'âge correspondait à 8 dents adultes étaient prépondérantes $(31 \%)$ par rapport aux femelles dont l'âge correspondait à 8 dents de lait $(8 \%)$ (Tableau 8). L'étude relationnelle avait montré l'absence de dépendance entre l'âge des femelles examinées et le résultat de l'échographie $(p>0,05)$. Néanmoins, en tenant compte de l'âge et des points de vente, l'effectif des femelles gestantes observées aux différents points de vente était fonction de l'âge (Tableau 9).

Tableau 8 : Effectif des femelles gestantes et non gestantes observées par rapport à l'âge

\begin{tabular}{lllll}
\hline & Femelles gestantes & Femelles non gestantes & Total & \\
\cline { 4 - 5 } & & & $\mathrm{n}$ & $\%$ \\
\hline 8dl & 10 & 14 & 24 & 8 \\
2DA & 22 & 17 & 39 & 14 \\
4DA & 11 & 18 & 29 & 10 \\
6DA & 19 & 18 & 37 & 13 \\
8DA & 44 & 45 & 89 & 31 \\
8ENT & 33 & 34 & 67 & 24 \\
Total & 139 & 146 & 285 & 100 \\
Moyenne & 23 & 24 & & \\
$\%$ & 49 & 51 & 100 & \\
\hline
\end{tabular}

Légende : $8 \mathrm{dl}-8$ dents de lait, 2DA- 2 dents d'adultes, 4DA- 4 dents d'adultes, 6DA- 6 dents d'adultes, 8DA- 8 dents d'adultes, 8 ENT - 8 dents d'adultes entamées

Tableau 9 : Effectif des femelles gestantes observées par rapport à l'âge aux différents points de vente

\begin{tabular}{lllll}
\hline & Marché L.D. KABILA & Camp VANGU & Rail KAMALONDO & Total \\
\hline 8dl & 3 & 0 & 7 & 10 \\
2DA & 11 & 8 & 3 & 22 \\
4DA & 4 & 2 & 5 & 11 \\
$6 \mathrm{DA}$ & 9 & 5 & 5 & 19 \\
8DA & 20 & 5 & 19 & 44 \\
8ENT & 13 & 13 & 7 & 33 \\
Total & 60 & 33 & 46 & 139
\end{tabular}

Légende : $8 \mathrm{dl}-8$ dents de lait, 2DA- 2 dents d'adultes, 4DA- 4 dents d'adultes, $6 \mathrm{DA}-6$ dents d'adultes, 8DA- 8 dents d'adultes, 8 ENT - 8 dents d'adultes entamées 


\section{Ngona et al. J. Appl. Biosci. La gestion de la reproduction caprine au travers des femelles vendues à}

différents points de vente de Lubumbashi en RDC.

\section{DISCUSSION}

Effectif des mâles et femelles : La proportion des mâles $(53 \%)$ et des femelles $(47 \%)$ exposés aux points de vente représentait respectivement 53 et $47 \%$ (Tableau 3). Chaque point de vente présentait 171 à 202 mâles exposés (Tableau 5). L"effectif observé aux différents points de vente était dépendante de sexe. Ceci traduisait la différence significative $(p<0,05)$ entre l'effectif mâle $(53 \%)$ et femelle $(47 \%)$ (Tableaux 3,4$)$. La sex-ratio était comprise comme une présentation de la proportion des mâles dans l'ensemble du troupeau. La sex-ratio permettait de mieux approcher la définition de la proportion des femelles. En effet, les chevrettes, géniteurs potentiels constituaient des femelles pour le renouvellement des chèvres en reproduction (Troedsson, 2002). Les femelles constituaient des principaux animateurs de la gestion de la reproduction pour garantir des bonnes performances de reproduction. Toutefois, une gestion rationnelle devrait écarter les mâles de la reproduction par suppression de la gamétogénèse. Ceci permettrait d'obtenir des bonnes performances de reproduction du troupeau. A cet effet, il convenait de relever que la consanguinité était un facteur d'altération (Khan et al., 2007).

Effectif des femelles présentes aux différents points de vente : Le pic (132 à 179 caprins exposés) était observé au mois de mai et les valeurs basales (71 à 126 caprins exposés) étaient observées en août pour la période observée (Fig. 2). Et particulièrement, les moyennes mensuelles avaient un pic en mai (52 caprins par jour) et le taux basal en août (31 caprins par jour) (Tableau VI). La disponibilité des femelles aux différents points de vente était fonction de mois et les différences entre les effectifs des femelles vendues pour les différents points de vente pour chaque mois étaient significatives $(p<0,05)$. Au regard de la plupart des chèvres qui étaient gestantes au mois de mars coïncidant avec les riches pâturages et qui présentaient un taux de conception élevé pendant la saison des pluies et faible en saison sèche, il y avait possibilité de préparer une

\section{CONCLUSION}

Notre étude a été réalisée à différents points de vente des chèvres sur 5416 caprins dont 2845 mâles (53\%) et 2571 femelles (47\%) de l'effectif total. L'âge était compris entre 7 et au-delà de 108 mois. Cette étude avait permis d'observer la gestion de la reproduction caprine au travers des femelles vendues aux marchés de Lubumbashi. Nos résultats avaient relevé $49 \%$ soit 139 cas de pertes de gestation sur 285 femelles ayant fait l'objet de constat de gestation. L'effectif des femelles réforme rationnelle. Cette dernière ne concernerait que la période au cours de laquelle les femelles seraient supposées non gravides. Nos résultats avaient montré qu'une forte proportion de femelles aux différents points de vente était fortement enregistrée au mois de mai (24\%) et faiblement enregistrée au mois d'août (14\%) (Tableau 7). En effet, cette période était marquée par une précarité alimentaire. Des meilleures conditions alimentaires étaient nécessaires pour une bonne croissance et par la suite pour une bonne prolificité (Delgadillo et al., 1997). Une restriction alimentaire aigüe montrait une réduction du taux d'ovulation, du taux d'ovulation multiple et une faible proportion des femelles gestantes (Nieto et al., 2006). Martinez-Rojero et al. (2006) avaient confirmé un effet significatif de la nutrition sur la prolificité.

Effectif des femelles gestantes: Le constat de gestation par échographie avait présenté des femelles non gestantes (51\%) et des femelles gestantes $(49 \%)$. Les femelles exposées au point de vente présentaient une importante proportion à un âge correspondait à 8 dents adultes (31\%) par rapport aux femelles dont l'âge correspondait à 8 dents de lait (8\%) (Tableau 8). En effet, la majorité des femelles gestantes ou non gestantes était âgée de 26 à 36 mois. L'âge moyen des femelles gestantes (38,8 mois) s'était avéré comparable à celui des femelles non gestantes (37,6 mois) (Ngona, 2008). Nos résultats avaient montré qu'il n'y avait pas de dépendance entre l'âge des femelles examinées et le résultat de l'échographie. Néanmoins, l'effectif des femelles gestantes observées aux différents points de vente était fonction de l'âge (Tableau 9). Des travaux effectués sur la chèvre locale avaient mis en évidence qu'à l'abattage, $44 \%$ des femelles étaient gestantes avec des gestations simples $(53,1 \%)$, gémellaires $(43,2 \%$ ) ou des triplets $(3,7 \%)$. Les pertes ainsi observées correspondaient à des produits dont $15 \%$ d'embryons et $85 \%$ de fœetus, et $47 \%$ de sexe femelle et $53 \%$ de sexe mâle (Ngona, 2008).

(gestantes et non gestantes) sur les différents points de vente traduisait une mauvaise gestion de la reproduction caprine à Lubumbashi. Toutefois, au regard des ventes mensuelles (787-1225 caprins), il était constaté que l'élevage caprin pouvait être rentable dans la ville de Lubumbashi. En perspectives, la reproduction caprine nécessiterait d'améliorer les conditions d'élevage de la chèvre locale et, par conséquent, les performances de reproduction à travers le maintien de l'index de prolificité, 
la détection aussi précoce que possible des femelles gestantes avant de les envoyer à l'abattoir. Aussi, une sensibilisation des éleveurs sur la capriculture serait

\section{BIBLIOGRAPHIE}

Delgadillo JA, Malpaux B, Chemineau P, 1997. La reproduction des caprins dans les zones tropicales et subtropicales. INRA Productions Animales, 10, 33-41

Khan MS, Ali, A, Hyder AU, Chatta Al, 2007. Effect of inbreeding on growth and reproduction traits of Beetal goats. Archiv für Tierzucht, Dummerstorf, 50: 197-203.

Martinez-Rojero RD, Hernandez-Ignacio J, HernandezHernandez $\mathrm{H}$, Michel-Aceves AC, ValenciaMendez J, 2006. Intrautrine artificial insemination in Creole goats with cooled semen. Agrociencia, 40: 71-76.

Ngona IA, 2008. Performances et facteurs d'influence de la reproduction de l'espèce caprine en milieu tropical. Thèse d'agrégation, Faculté de Médecine Vétérinaire, Université de Lubumbashi. 165 pp.

Ngona IA, Beduin JM, Khang'Maté ABF, Hanzen C, 2012. Étude descriptive des caractéristiques morphométriques et génitales de la chèvre de Lubumbashi en République démocratique du nécessaire. Enfin, il faudrait une bonne conduite d'élevage pour pallier les pertes de gestation et mettre à profit la prolificité de la chèvre.

Congo. Revue d'élevage et de médecine vétérinaire des pays tropicaux, $65: 75-79$.

Nieto CAR, Morales JU, Vasquez HG, Gomez MOD, Andrade BMR, 2006. The influence of feeding level on the reproductive activity of Mexican mature goats during the reproductive season. Tecnica Pecuaria en Mexico, 44: 399-406.

Troedsson MHT, 2002. Alterations in sexual function. Smith BP (Editor). $3^{\text {th }}$ ed. Mosby : Saint Louis. Missouri, US. pp 1735

Vandenput S 2006. Contribution à la satisfaction des besoins économiques et alimentaires de la population lushoise par le développement d'un élevage caprin intégré aux activités agricoles péri-urbaines. Projets interuniversitaires Ciblés en matière de Coopération au développement, Université de Liège, Faculté Universitaire des Sciences Agronomiques de Gembloux, Université de Lubumbashi, 2006.

Zarrouk A, Souilem O, Drion PV, Beckers JF, 2001. Caractéristiques de la reproduction de l'espèce caprine. Annales de Médecine Vétérinaire, Université de Liège, $145: 98-105$ 